\title{
Electrophysiological correlates of the efficient detection of emotional facial expressions.
}

\section{$\operatorname{AUTHOR}(\mathrm{S})$ :}

Sawada, Reiko; Sato, Wataru; Uono, Shota;

Kochiyama, Takanori; Toichi, Motomi

\section{CITATION:}

Sawada, Reiko ... [et al]. Electrophysiological correlates of the efficient detection of emotional facial expressions.. Brain research 2014, 1560: 60-72

\section{ISSUE DATE:}

2014-04-29

URL:

http://hdl.handle.net/2433/187077

\section{RIGHT:}

(c) 2014 Elsevier B.V.; この論文は著者最終稿です。内容が印刷版と異な ることがありますので、引用の際には出版社版をご確認ご利用くださ い。 This is the Accepted Author Manuscript. Please cite only the published version. 


\title{
Electrophysiological correlates of the efficient detection of emotional facial expressions
}

\author{
Reiko Sawada ${ }^{a}$, Wataru Sato ${ }^{\mathrm{a}}$, Shota Uono ${ }^{\mathrm{b}}$, Takanori Kochiyama ${ }^{\mathrm{a}}$, Motomi Toichi ${ }^{\mathrm{b}}$ \\ ${ }^{a}$ Primate Research Institute, Kyoto University, \\ ${ }^{b}$ Faculty of Human Health Science, Graduate School of Medicine, Kyoto University
}

\begin{abstract}
Behavioral studies have shown that emotional facial expressions are detected more rapidly and accurately than are neutral expressions. However, the neural mechanism underlying this efficient detection has remained unclear. To investigate this mechanism, we measured event-related potentials (ERPs) during a visual search task in which participants detected the normal emotional facial expressions of anger and happiness or their control stimuli, termed "anti-expressions," within crowds of neutral expressions. The anti-expressions, which were created using a morphing technique that produced changes equivalent to those in the normal emotional facial expressions compared with the neutral facial expressions, were most frequently recognized as emotionally neutral. Behaviorally, normal expressions were detected faster and more accurately and were rated as more emotionally arousing than were the anti-expressions. Regarding ERPs, the normal expressions elicited larger early posterior negativity (EPN) at 200-400 ms compared with anti-expressions. Furthermore, larger EPN was related to faster and more accurate detection and higher emotional arousal. These data suggest that the efficient detection of emotional facial expressions is implemented via enhanced activation of the posterior visual cortices at $200-400 \mathrm{~ms}$ based on their emotional significance.
\end{abstract}

Keywords: Anti-expression, Arousal, Emotional facial expression, Early posterior negativity (EPN), Visual search.

\section{Introduction}

Emotional facial expressions are proposed to have evolved as indispensable communication media for the survival of human ancestors (Darwin, 1872). Rapid and accurate communication via emotional facial expressions would have facilitated immediate sharing of biologically significant information, such as that about predators or food.

Consistent with this idea, several behavioral studies have demonstrated efficient detection of the emotional facial expressions of others using a visual search paradigm. The visual search paradigm has been applied successfully to demonstrate the ability of the human visual system to detect socially important signals in the environment. In previous studies, photographs or drawings of facial expressions were lined up, and participants were asked to respond regarding the existence of different expressions. The results showed that the reaction time (RT) for and accuracy of detecting an emotional face (e.g., angry and happy) in a crowd of neutral faces were shorter and greater, respectively, than were those for a neutral face in a crowd of emotional faces (Hansen and Hansen, 1988, Öhman et al., 2001 and Williams et al., 2005). Thus, the detection of emotional facial expressions is more effective than the detection of neutral expressions.

It remains controversial whether the rapid detection of emotional faces could be derived from emotional or visual factors (cf. Cave and Batty, 2006) because emotional and neutral facial expressions are different not only in their emotional significance but also in the change in visual features (e.g., oblique eyebrows in angry expressions versus horizontal eyebrows in neutral expressions). Several behavioral studies have shown that oblique lines were 
detected more quickly than horizontal lines (Sagi and Julesz, 1986), suggesting that changes in physical features play an important role in the rapid detection of emotional facial expressions. However, a recent behavioral study investigated this issue by manipulating the visual features of photographic facial stimuli (Sato and Yoshikawa, 2010). The researchers presented normal emotional facial expressions (anger and happiness) or control stimuli, termed "anti-expressions" (Sato and Yoshikawa, 2009; for different use of this term, see Skinner and Benton, 2010 and Skinner and Benton, 2012), within crowds of neutral expressions. Anti-expressions were created from photographs of emotional facial expressions by using computer-morphing techniques to contain a degree of visual changes equivalent to those in emotional facial expressions compared with neutral facial expressions; however, the anti-expressions were recognized as neutral expressions in free categorical labeling (Sato and Yoshikawa, 2009). This method enabled us to determine whether the rapid detection of emotional facial expressions was attributed to emotional significance or to visual features. Therefore, it was suggested that anti-expressions are promising control stimuli for investigating the effects of emotional significance on the efficient detection of emotional facial expressions (Sato and Yoshikawa, 2009). The previous study showed that the RTs for detecting emotional facial expressions were shorter than those for detecting anti-expressions, indicating that emotional facial expressions are efficiently detected because of their emotional, not their visual, elements (Sato and Yoshikawa, 2010).

Although these accumulating behavioral data have confirmed the rapid and accurate detection of emotional facial expressions, its neural mechanism remains unclear. To measure the brain activity underlying such rapid psychological processing, event-related potentials (ERPs) would be one of the most appropriate tools because of their high temporal resolution. A few recent studies have reported ERP data in visual search tasks with facial stimuli (Feldmann-Wüstefeld et al., 2011 and Weymar et al., 2011). However, these studies focused only on differences among emotional categories of targets (i.e., angry versus happy expressions). Thus, no reported study has compared the ERP components for detecting emotional versus emotionally neutral facial targets within neutral distractors. Furthermore, the influence of emotional and visual factors on ERP activity related to the detection of emotional versus emotionally neutral facial targets within neutral distractors has not been examined. There is also no report on the relationships between detection performance or emotional ratings for emotional expressions and ERP activities; such information would be of importance in understanding the functional significance of the influence of emotion on ERP components (Olofsson et al., 2008).

There appear to be two ERP candidates for the electrophysiological correlate of rapid detection of emotional facial expressions. The first is the negative deflection at about $200-400 \mathrm{~ms}$ in the bilateral posterior cortices, referred to as early posterior negativity (EPN) (Schupp et al., 2003). Several ERP studies showed that greater EPN was elicited in response to emotional than to neutral facial expressions (e.g., Balconi and Pozzoli, 2003, Sato et al., 2001 and Schupp et al., 2004) and in response to emotional than to neutral scenes (e.g., Junghöfer et al., 2001, Schupp et al., 2003 and Wiens et al., 2011; for a review, see Olofsson et al., 2008). A previous study also showed that greater EPN was elicited by emotional rather than by neutral scenes, even when some visual factors (e.g., luminance and spatial frequency) were controlled (Wiens et al., 2011). This study also showed that greater EPN was related to higher ratings of emotional arousal for scenery stimuli. Some researchers have suggested that the enhanced EPN for emotional stimuli may reflect heightened perceptual or attentional processing of the stimuli (e.g., Sato et al., 2001 and Schupp et al., 2003). These empirical and theoretical data on EPN are consistent with 
behavioral data indicating the rapid detection of emotional compared with neutral facial expressions, even when controlling for visual factors, and their relevance to emotional arousal (Sato and Yoshikawa, 2010). Thus, we hypothesized that EPN would be the primary candidate for the neural correlate of the efficient detection of emotional facial expressions.

The second candidate is the negative deflection at around $200-400 \mathrm{~ms}$ in the posterior cortices contralateral to the target presentation, referred to as N2-posterior-contralateral (N2pc) (Luck and Hillyard, 1994). Several previous ERP studies have shown that greater N2pc was elicited in response to the target stimuli in a visual search paradigm (e.g., Luck and Hillyard, 1994 and Woodman and Luck, 1999). Some recent studies tested emotional facial expressions as targets during the detection of emotional targets in a visual search task. These studies showed that greater N2pc was elicited by targets detected more rapidly than by those detected later (Feldmann-Wüstefeld et al., 2011 and Weymar et al., 2011). However, these previous studies did not compare emotional with neutral facial expression targets. Regarding the effects of visual versus emotional factors, a study reported that visual factors, such as local features, did not modulate N2pc in response to emotional facial expressions (Weymar et al., 2011). In contrast, another study reported that visual factors were related to N2pc activity in response to emotional facial expressions (Brosch et al., 2011). Moreover, no reported study has investigated the relationship between N2pc amplitude and emotional arousal. Thus, although the evidence is relatively scarce, we predicted that N2pc would be the secondary candidate related to the detection of emotional facial expressions.

To investigate the neural mechanism underlying the efficient detection of emotional facial expressions, we measured ERP during a visual search task. Our primary aim was to clarify the electrophysiological correlates of emotional processing, and not processing of local visual features, of emotional facial expressions. The normal expressions and anti-expressions of anger or happiness were presented as targets within crowds of neutral expressions (Fig. 1). To investigate the emotional processes related to target detection, we required participants to rate the subjectively experienced arousal and valence (cf. Lang et al., 1998). We also tested stimulus familiarity and naturalness as possible confounding factors (e.g., Tong and Nakayama, 1999). Based on the previously mentioned evidence, we made three predictions. First, enhanced EPN/N2pc would be induced in response to normal expressions compared with anti-expressions. Second, the greater EPN/N2pc activity would be related to shorter RTs and increased accuracy. Third, the greater EPN/N2pc activity would be related to higher emotional arousal ratings.

(a)

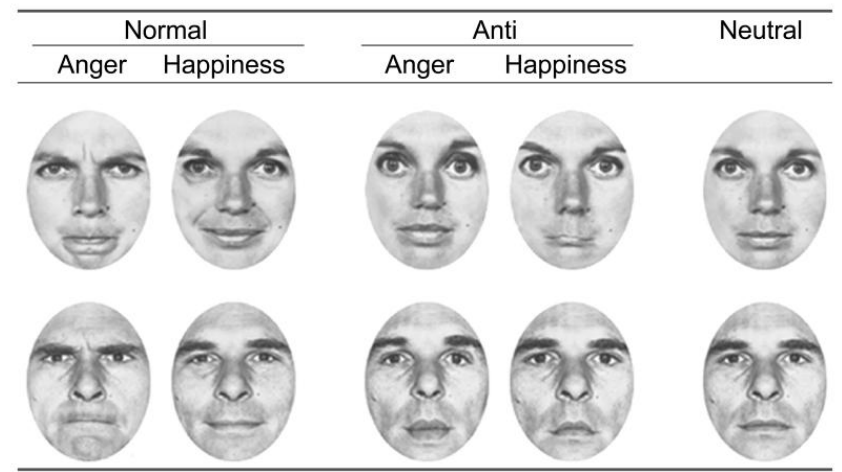

(b)

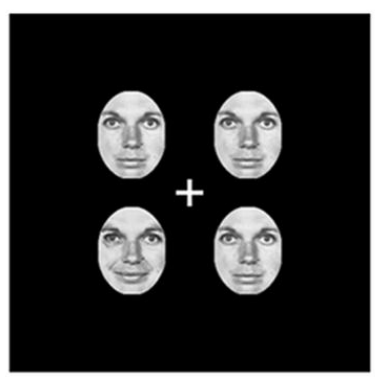

Fig. 1. Stimuli (a). Targets (normal expressions and anti-expressions of anger and happiness) and distractors (neutral expressions). Examples of stimulus arrays (b). 


\section{Results}

\subsection{RT and accuracy}

Mean (with SE) RTs and accuracy rates are shown in Fig. 2. To investigate the effect of stimulus facial expressions on detection performance, the RTs and accuracy rates were analyzed using three-way repeated-measures analyses of variance (ANOVAs) with type (normal expression and anti-expression), emotion (anger and happiness), and visual field (left and right) as factors.

(a)

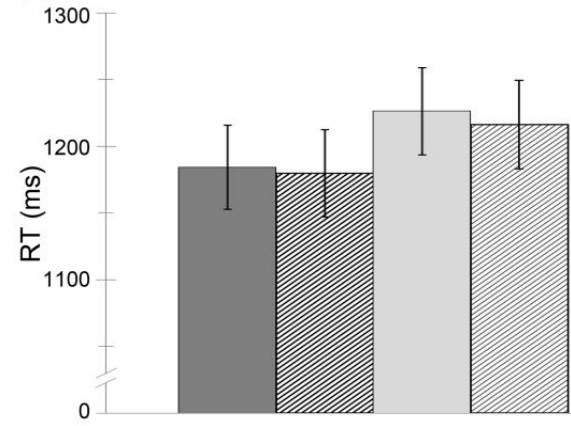

(b)

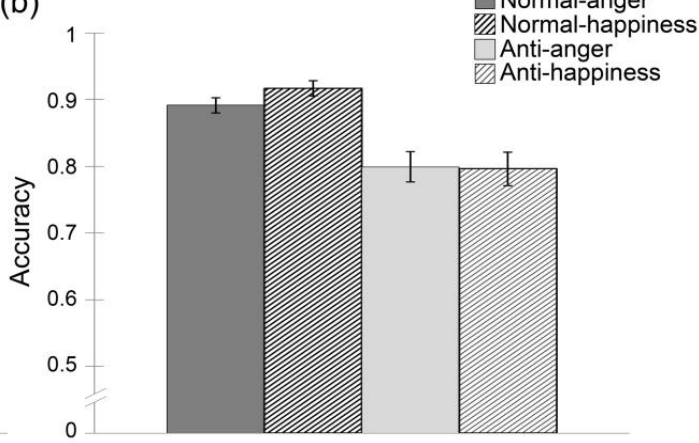

Fig. 2. Mean (with $S E$ ) reaction time (RT) (a) and accuracy (b) for each stimulus condition. The visual field factor is collapsed.

For RTs, the results revealed significant main effects of type $(F(1,19)=29.96, p<0.001)$ and visual field $(F(1$, $19)=4.74, p<0.05)$, and a significant interaction of typexemotion $\times$ visual field $(F(1,19)=6.14, p<0.05)$. Follow-up analyses for the three-way interaction revealed that the simple effect of type, indicating shorter RTs for normal expressions than for anti-expressions, was significant for all emotion $\times$ visual field conditions (anger-left: $F(1$, $76)=18.39, p<0.001$; anger-right: $F(1,76)=7.44, p<0.05$; happiness-left: $F(1,76)=6.67, p<0.05$; happiness-right: $F(1,76)=20.19, p<0.001)$. The simple effect of emotion was significant for anti-expression presented in the left visual field $(F(1,76)=7.10, p<0.05)$, indicating shorter RTs for anti-happiness than for anti-anger, but this was not significant in other conditions $(F(1,76)<1.60, p>0.10)$. The simple effect of visual field was significant for anti-anger $(F(1,76)=8.41, p<0.01)$, indicating shorter RTs for anti-anger presented in the right visual field than in the left visual field, but it was not significant in other conditions $(F(1,76)<3.60, p>0.05)$.

In terms of accuracy, the ANOVA revealed significant main effects of type $(F(1,19)=26.62, p<0.001)$ and visual field $(F(1,19)=26.08, p<0.001)$, and a significant interaction of typexvisual field $(F(1,19)=7.30, p<0.05)$. Follow-up analyses for the two-way interaction revealed that the simple effect of type, indicating higher accuracy for normal expressions than for anti-expressions, was significant for both visual field conditions (left: $F(1$, $38)=31.77$; right: $F(1,38)=15.56$, both $p<0.01)$. The simple effect of visual field, indicating higher accuracy when the stimuli were presented in the right than in the left visual field, was significant for both stimulus conditions (normal: $F(1,38)=14.93$; anti-expression: $F(1,38)=21.72$, both $p<0.01$ ).

In summary, the RT and accuracy data showed that normal expressions were detected more rapidly and more accurately than were anti-expressions. There was no evidence of a speed-accuracy trade-off phenomenon.

\subsection{Rating}

The mean (with $S E$ ) ratings for four items (arousal, valence, familiarity, and naturalness) are shown in Table 1. 
To investigate differences in emotional responses to target stimuli, each rating for target facial stimuli was analyzed using a repeated-measures ANOVA with type and emotion as factors.

Table 1. Mean (with $S E$ ) ratings for each facial expression.

\begin{tabular}{ccccccc}
\hline \multirow{2}{*}{ Item } & \multicolumn{2}{c}{ Normal } & & \multicolumn{2}{c}{ Anti } & \multirow{2}{*}{ Neutral } \\
\cline { 2 - 3 } \cline { 5 - 6 } & Anger & Happiness & & Anger & Happiness & \\
\hline Arousal & $7.03(0.22)$ & $5.90(0.31)$ & & $4.93(0.21)$ & $4.83(0.20)$ & $4.03(0.25)$ \\
Valence & $2.60(0.21)$ & $7.03(0.26)$ & & $4.60(0.24)$ & $4.18(0.13)$ & $5.00(0.21)$ \\
Familiarity & $2.38(0.29)$ & $7.05(0.27)$ & & $4.25(0.28)$ & $3.98(0.21)$ & $5.03(0.28)$ \\
Naturalness & $3.28(0.39)$ & $6.35(0.40)$ & & $4.38(0.37)$ & $4.08(0.34)$ & $6.83(0.33)$ \\
\hline
\end{tabular}

With respect to arousal, the main effects of type $(F(1,19)=45.80, p<0.001)$ and emotion $(F(1,19)=6.61$, $p<0.05)$ and the interaction of typexemotion $(F(1,19)=5.17, p<0.05)$ were significant. The follow-up analysis for the interaction showed that the simple effect of type, indicating higher arousal ratings for normal expressions than for anti-expressions, was significant for both anger $(F(1,38)=68.96, p<0.001)$ and happiness $(F(1,38)=7.82$, $p<0.05)$. The simple effect of emotion was also significant for normal expressions $(F(1,38)=8.02, p<0.05)$, indicating higher arousal ratings for normal-anger than for normal-happiness, but it was not significant for anti-expressions $(F(1,38)=0.17, p>0.10)$.

In terms of valence, the main effects of type $(F(1,19)=5.49, p<0.05)$ and emotion $(F(1,19)=111.56, p<0.001)$ and the interaction of typexemotion $(F(1,19)=125.81, p<0.001)$ were significant. Further examination of the two-way interaction showed that the simple effect of type was significant for both expressions (anger: $F(1$, $38)=46.77$, happiness: $F(1,38)=110.04$, both $p<0.001)$, indicating lower valence ratings for normal-anger than for anti-anger and higher valence ratings for normal-happiness than for anti-happiness. The simple effect of emotion was significant for normal $(F(1,38)=156.19, p<0.001)$ and for anti-expressions $(F(1,38)=4.53, p<0.05)$, indicating lower valence ratings for normal-anger than for normal-happiness and higher valence ratings for anti-anger than for anti-happiness.

With regard to familiarity, the main effects of emotion $(F(1,19)=135.48, p<0.001)$ and the interaction of typexemotion $(F(1,19)=79.96, p<0.001)$ were significant. The follow-up analysis for the interaction showed that the simple effect of type was significant for both expressions (anger: $F(1,38)=18.70$; happiness: $F(1,38)=68.92$, both $p<0.001$ ), indicating lower familiarity for normal-anger than for anti-anger and higher familiarity for normal-happiness than for anti-happiness. The simple effect of emotion was significant for normal expressions $(F(1$, $38)=165.65, p<0.001$ ), indicating lower familiarity for normal-anger than for normal-happiness, but it was not significant for anti-expressions $(F(1,38)=0.82, p>0.10)$.

With respect to naturalness, the main effects of emotion $(F(1,19)=22.91, p<0.001)$ and the interaction of typexemotion $(F(1,19)=28.30, p<0.001)$ were significant. In terms of the interaction, the simple effect of type was significant for happy expressions $(F(1,38)=24.36, p<0.001)$, indicating higher naturalness ratings for normal-happiness than for anti-happiness, but this was not the case for anger $(F(1,38)=4.08, p>0.05)$. The simple effect of emotion was significant for normal expressions $(F(1,38)=38.79, p<0.001)$, indicating higher naturalness 
ratings for normal-happiness than for normal-anger, but it was not significant for anti-expressions $(F(1,38)=0.72$, $p>0.10)$.

In short, the results of the ratings for targets revealed that only the arousal ratings showed a consistent effect of type (normal versus anti-expression) across angry and happy expressions, which correspond to the results of the RTs and accuracy.

\subsection{Rating- $R T$ and rating-accuracy relationships}

To investigate the relationships between subjective emotional reactions and detection performance in response to target stimuli, a multiple regression analysis was conducted with the single rating as an independent variable and RT or accuracy as a dependent variable.

For the rating-RT relationship, the results showed that arousal was significantly related to RT $(t(59)=4.02$, $p<0.001)$, indicating that higher arousal ratings were related to shorter detection RTs. Other ratings were not significantly related to RT (valence: $t(59)=1.30$; familiarity: $t(59)=1.65$; naturalness: $t(59)=1.33$, all $p>0.10$ ).

In terms of accuracy, the multiple regression analysis showed that arousal was significantly related to accuracy $(t(59)=2.89, p<0.01)$, indicating that higher arousal ratings were related to more accurate detection. Familiarity $(t(59)=2.13, p<0.05)$ and naturalness $(t(59)=2.01, p<0.05)$ were also related to accuracy, indicating that higher familiarity and naturalness were related to more accurate detection. In contrast, valence was not related to accuracy $(t(59)=1.45, p>0.10)$.

In summary, higher arousal was consistently related to more rapid and more accurate detection.

\subsection{ERP}

Fig. 3 and Supplementary Fig. 1 show the grand average ERPs recorded at the P7 and P8 electrodes. The ERP data were analyzed using a 3D space-time SPM approach (Kilner and Friston, 2010 and Litvak et al., 2011) to appropriately process the problem of multiple comparisons over latencies and/or electrodes. The data were analyzed with repeated-measures ANOVA models with type, emotion, and visual field as factors.

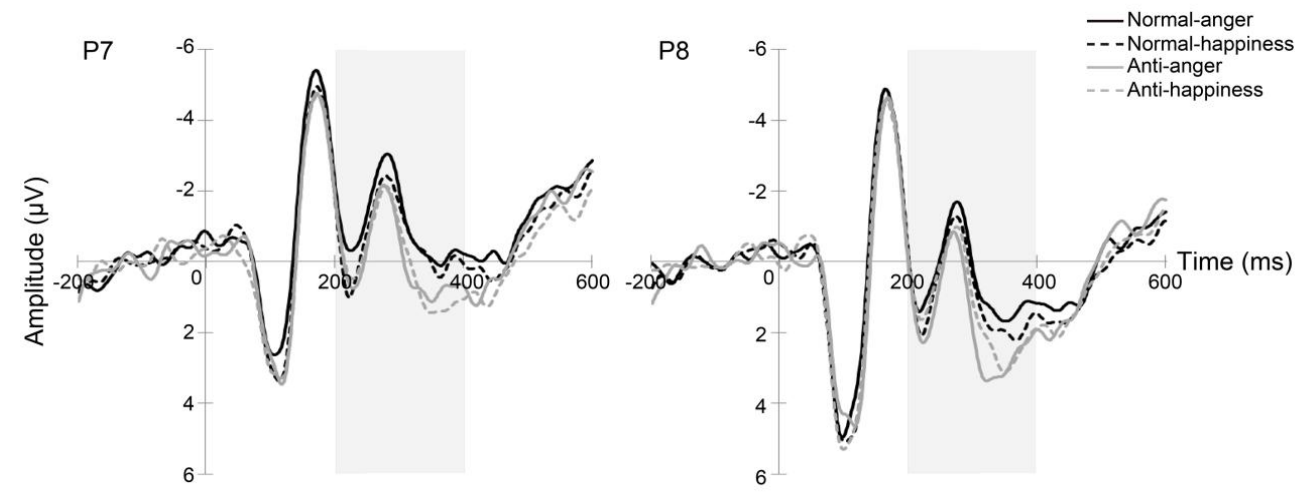

Fig. 3. Grand average ERP waveforms at the P7 and P8 electrodes. Data for each stimulus condition are shown. The visual field factor is collapsed. The time window of interest (200-400 ms) is shaded.

To analyze EPN, the main effect of type, comparing normal versus anti-expressions, was tested. The results showed a significant negative deflection during 200-400 ms at the P7 (peak: $346 \mathrm{ms,}$ 
$Z=3.27, p<0.01$, corrected; time extent: $302-358 \mathrm{~ms}$ ) and P8 (peak: $346 \mathrm{~ms}, Z=3.45, p<0.01$, corrected; peak: $302 \mathrm{~ms}, Z=3.08, p<0.05$, corrected; time extent: $284-360 \mathrm{~ms}$ ) electrode locations (Fig. 4$)$. The topographic map of $p$-values indicated that a cluster of bilateral posterior regions was significantly activated. No significant effect of type was observed for any other space-time regions. We also conducted analyses for other main effects or interactions in the whole space-time regions but found no significant results.

(a)
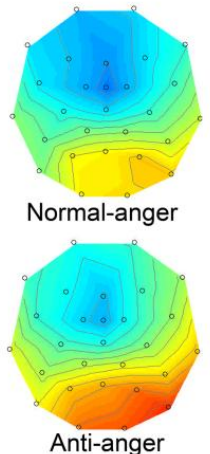

(c)

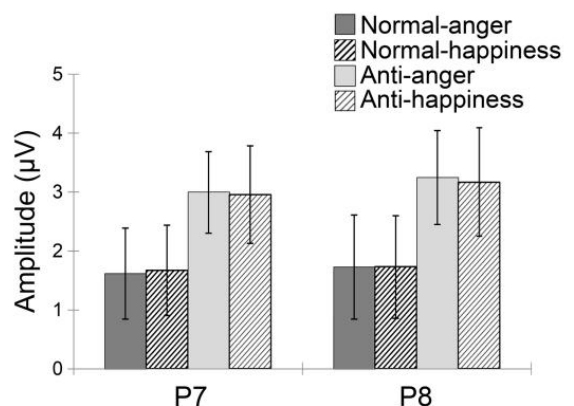

(b)

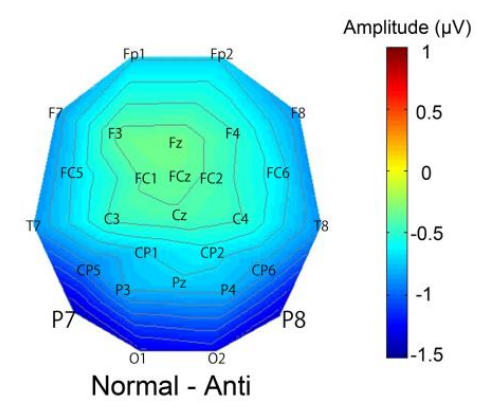

(d)

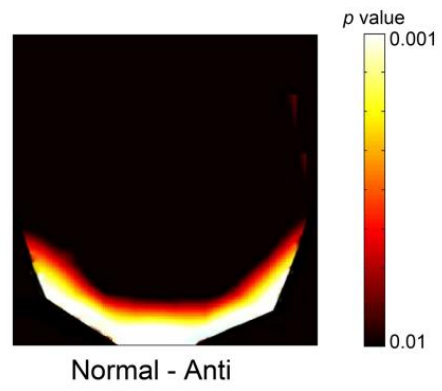

Fig. 4. ERPs at $346 \mathrm{~ms}$ showing EPN for normal expressions versus anti-expressions. (a) Topographic map of amplitude for each stimulus condition. (b) Topographic map of differential amplitudes between normal expression and anti-expression conditions. (c) Mean (with SE) amplitudes at P7 and P8 electrodes. (d) Topographic map of p-values for the contrast between normal expressions and anti-expressions (uncorrected $\mathrm{p}<0.01$ for display purposes).

To examine N2pc, the interaction among type, visual field and electrode were analyzed. There was no significant activity within our space-time region of interest (ROI). With a more liberal height threshold $(p<0.05$, uncorrected), activation was found at the P8 electrode location (peak: $244 \mathrm{~ms} ; Z=2.09$ ). No significant interactions were observed for other space-time regions. Moreover, we conducted analyses for other main effects or interactions in the whole space-time regions but found no significant results.

To confirm these results and to complement the bin-based approach of SPM (Kilner and Friston, 2010), additional ROI analyses were conducted by calculating mean amplitudes during 200-400 ms at the P7 and P8 electrodes. Four-way repeated-measures ANOVAs with type, emotion, visual field, and electrode as factors were conducted. The main effects of type $(F(1,19)=13.98, p<0.005)$ and visual field $(F(1,19)=17.16, p<0.005)$ and the interaction of visual field $\times$ electrode $(F(1,19)=8.33, p<0.01)$ were significant. Other main effects and interactions were not significant $(F(1,19)<4.07, p>0.10)$. These results indicate larger negativities for normal expressions than for anti-expressions. Follow-up analyses for the interaction of visual fieldxelectrode indicated larger negativities 
for the targets in the right versus the left visual field at the left versus the right electrodes $(F(1,38)>7.20, p<0.05)$. Because several previous studies analyzed EPN/N2pc at different adjacent electrodes (e.g., Weymar et al., 2011), we also analyzed the ERP data at $\mathrm{O} 1 / \mathrm{O} 2$ and $\mathrm{P} 3 / \mathrm{P} 4$ in the same way. The results showed patterns similar to those for the $\mathrm{P} 7$ and $\mathrm{P} 8$ electrodes, showing the significant main effect of type and hemisphere of electrodes $(F(1$, $19)>13.84, p<0.01)$ and the interaction of visual field $\times$ hemisphere of electrodes $(F(1,19)>8.91, p<0.01)$, but no other significant main effects or interactions were found $(F(1,19)<3.53, p>0.05)$. The results showed no difference in the N2pc amplitude between normal and anti-expressions.

In summary, both the SPM and mean amplitude analyses revealed that normal expressions enhanced EPN compared with anti-expressions, whereas there was no significant difference between normal and anti-expressions in $\mathrm{N} 2 \mathrm{pc}$.

\section{5. $R T-$-, accuracy-, and rating-ERP relationships}

To test our predictions that the efficient detection and emotional significance of target stimuli were related to ERP components, the ERP data were analyzed further using regression models with RT, accuracy, or rating data as independent variables in a SPM approach.

For EPN, ERPs averaged across the left and right visual fields were used as the dependent variable. For the regression analysis with RT, the results revealed a significant negative relationship between RT and the absolute amplitude of negative deflections during 200-400 ms at the electrode sites P7 (peak: $356 \mathrm{~ms}, Z=3.32, p<0.01$, corrected; peak: $388 \mathrm{~ms}, Z=2.96, p<0.05$, corrected; time extent: $344-400 \mathrm{~ms}$ ) and P8 (peak: $354 \mathrm{~ms}, Z=3.77$, $p=0.001$, corrected; peak: $314 \mathrm{~ms} ; Z=3.30, p<0.01$, corrected; time extent: $290-372 \mathrm{~ms}$ ) ( Fig. 5(a)). The topographic map of $p$-values revealed that a cluster in the bilateral posterior cortices was significant. The result showed that larger EPN was associated with faster detection. There was no other significant cluster for any space-time regions.

The results of the regression analysis with accuracy revealed a significant positive relationship between accuracy and the absolute amplitude of the negative deflections at 200-400 ms at electrode sites P7 (peak: $354 \mathrm{~ms}$ and $392 \mathrm{~ms}, Z=3.29$ and 3.16, $p<0.01$, corrected; time extent: $342-400 \mathrm{~ms}$ ) and P8 (peak: $352 \mathrm{~ms}, Z=2.91, p<0.05$, corrected; time extent: $346-358 \mathrm{~ms}$ ) (Fig. 5(b)). The topographic map of $p$-values revealed that a cluster in the bilateral posterior cortices was significant. The results showed that greater EPN was associated with more accurate detection. There was no other significant cluster for any space-time regions.

For the analysis with arousal ratings as an independent variable, a significant positive relationship was found between the arousal rating and the absolute amplitude of negative deflection during 200-400 ms at P7 (peak: $346 \mathrm{~ms}, Z=2.56, p=0.05$, corrected, time extent: $330-358 \mathrm{~ms}$ ) and P8 (peak: $370 \mathrm{~ms}, Z=3.83, p<0.01$, corrected; peak: $280 \mathrm{~ms}, Z=2.82, p<0.05$, corrected; time extent: 270-394 ms) (Fig. 5(c)). The topographic $p$-value map again showed a significant cluster in the bilateral posterior regions. The result showed larger EPN associated with higher emotional arousal. No significant cluster was observed for any other space-time regions. We also conducted analyses using valence, familiarity, and naturalness ratings as independent variables but the data revealed no significant activity in any space-time regions. 


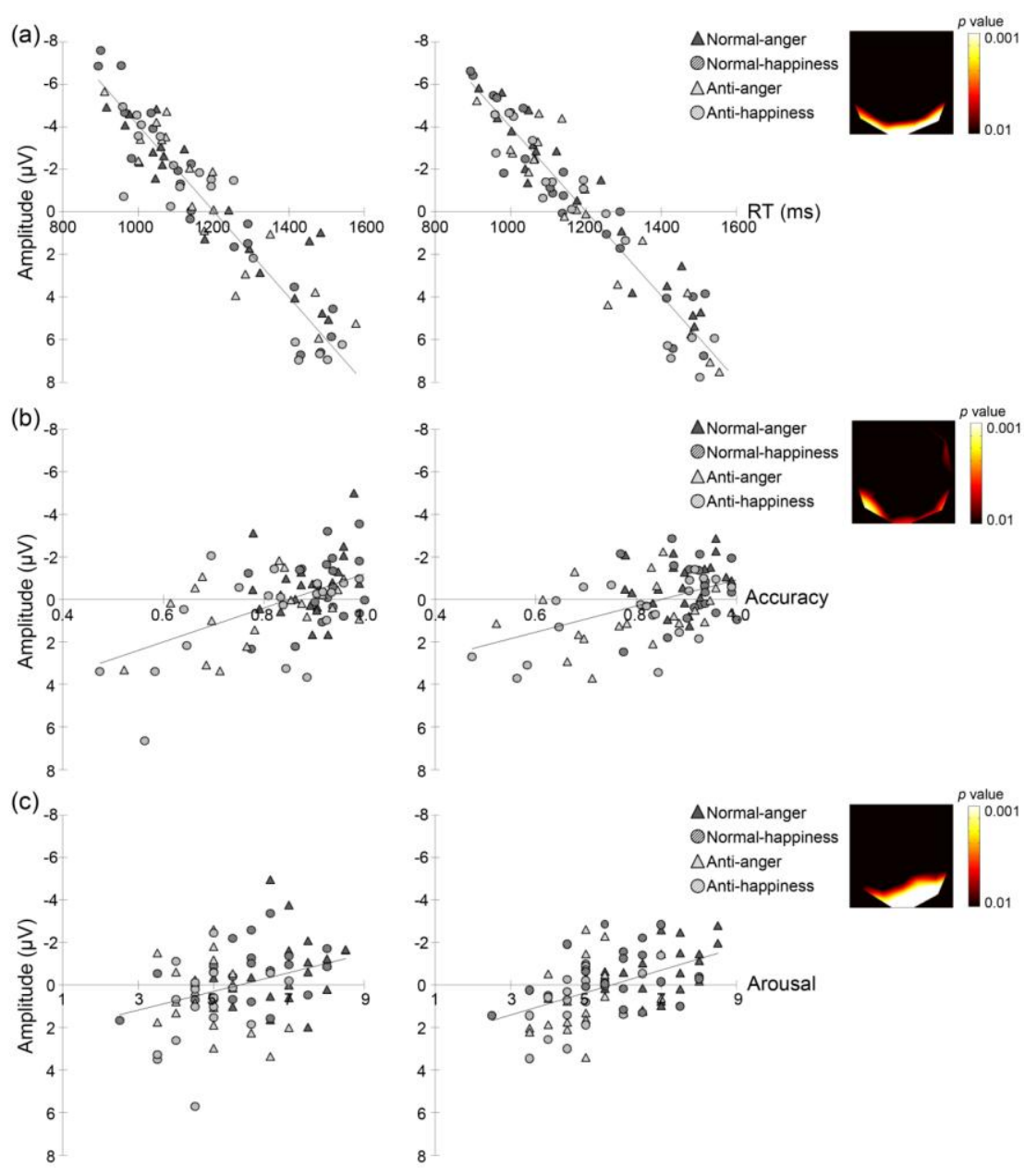

Fig. 5. Relationships between behavioral measures and EPN. Data for reaction time (RT) (a), accuracy (b), and arousal rating (c) are shown. The scatter plots and regression lines indicate the relationships between the behavioral measures and adjusted EPN amplitudes (after covarying out the participant effect) at the P7 (left) and P8 (right) electrode locations. The data were extracted from the activation foci in regression analyses. Topographic p-value maps show the effects of behavioral measures in regression analyses at $350 \mathrm{~ms}$ (uncorrected $\mathrm{p}<0.01$, only for display purposes).

For N2pc, differential ERPs between the contralateral and ipsilateral to the target presentation were analyzed. For the regression analysis with the RT as an independent variable, the results revealed a significant negative relationship between RT and the absolute amplitude of negative deflection of N2pc during 200-400 ms at the P8 electrode site (peak: $338 \mathrm{~ms}, Z=3.28, p<0.01$, corrected; time extent: $324-348 \mathrm{~ms}$ ). However, the topographic map of $p$-values indicated that a significant cluster extended into the central and frontal regions in the right hemisphere. Furthermore, no significant cluster was found during 200-400 ms at the P7 electrode, even with a more liberal height threshold ( $p<0.05$, uncorrected). Thus, these results should be regarded as a partial support, at best, for N2pc involvement in target detection. There was no other significant activation. The analyses using accuracy and all of the ratings as independent variables revealed no significant cluster in any space-time regions.

To confirm the results of the SPM approach, ROI-based regression analyses were conducted using mean amplitudes during 200-400 ms. The analyses of EPN revealed that larger negative deflections at 200-400 ms were 
significantly related to shorter RTs $(t(59)=2.31, p<0.05)$, higher levels of accuracy $(t(59)=1.85, p<0.05)$, and higher arousal ratings $(t(59)=2.57, p<0.05)$. There was no significant relationship between ERP activity and other ratings $(t(59)<0.88, p>0.10)$. For the analyses of $\mathrm{N} 2 \mathrm{pc}$, results showed that higher differential ERPs were significantly related to shorter RTs $(t(59)=2.20, p<0.05)$, but not to accuracy $(t(59)=0.38, p>0.10)$ and all ratings $(t(59)<1.41$, $p>0.10)$.

In summary, the SPM and mean amplitude analyses consistently showed that enhanced EPN was related to more rapid and accurate detection and higher arousal ratings for facial expressions. The relationships between N2pc and these behavioral measures were shown only partially.

\section{Discussion}

Our behavioral data of detection performance showed that the normal expressions of anger and happiness were detected faster and more accurately than were anti-expressions. The emotional rating results showed the corresponding pattern, in which normal expressions of anger and happiness were rated as more emotionally arousing than were their anti-expressions. Regression analyses confirmed the relationship between ratings and detection performance, showing that heightened emotional arousal was related to shorter RTs and higher accuracy for detecting expressions. These results are consistent with and also extend those of a previous study (Sato and Yoshikawa, 2010).

More importantly, our analyses of ERP data consistently revealed that such behavioral performance was related to EPN. First, the repeated-measures ANOVA of the ERPs showed that the normal expressions elicited greater EPN than did the anti-expressions. The results of our preliminary experiment showed that anti-expressions were most frequently labeled as neutral (see Section 4), which is consistent with a previous study (Sato and Yoshikawa, 2009). Therefore, these ERP results suggest that the emotional facial targets elicited greater EPN than did emotionally neutral facial targets. This result is consistent with the results of several previous ERP studies that reported greater EPN for emotional facial expressions (e.g., Sato et al., 2001) and emotional scenes (e.g., Junghöfer et al., 2001) than for neutral stimuli. Our results extend these observations, suggesting that emotional facial expressions elicited greater EPN during a visual search experiment compared with neutral ones and that their patterns correspond to behavioral data showing more rapid and more accurate detection of emotional than of neutral facial targets. Furthermore, because the anti-expressions contain changes in visual features comparable to those in normal expressions compared with neutral expressions, our results suggest that the increased EPN associated with the detection of emotional facial expressions is not attributable to visual factors. This is consistent with the results of a recent ERP study reporting that greater EPN was elicited by emotional than by neutral scenes, even after controlling for visual factors, including compositional complexity, spatial frequency, luminance, and contrast (Wiens et al., 2011). Our results extend these findings by indicating that differences of feature distortions, such as oblique lines and curves, do not account for increased EPN in response to emotional versus neutral stimuli. In summary, our results suggest that greater EPN is elicited during the detection of emotional than neutral facial expressions, and this difference is attributable to emotional and not to visual factors.

Second, multiple regression analyses of the ERP data revealed directly that increased EPN was related to faster and more accurate detection of the facial targets. These results, as well as the aforementioned correspondence 
of the results of factorial analyses between behavioral and ERP data, suggest that EPN is related to the rapid and accurate detection of facial expressions. These results provide an empirical support for previous suggestions of a relationship between EPN and detection performance. For example, it has been proposed that enhancement of EPN may reflect the tagging of stimuli for further processing of sensory encoding (Schupp et al., 2004) or the greater allocation of attentional resources to emotional than to neutral scenes (Schupp et al., 2003). It has been also proposed that greater EPN may reflect enhanced conscious awareness of emotional than that of neutral facial expressions (Sato et al., 2001). Furthermore, our results are consistent with another line of ERP research investigating conscious awareness of non-emotional stimuli. Several studies have reported that the negative deflection at the posterior cortices during 200-400 ms was greater in response to consciously seen stimuli than to non-seen stimuli (e.g., Genetti et al., 2009; for a review, see Koivisto and Revonsuo, 2010). Together with these data, our results suggest that greater EPN reflects neural processing related to the rapid and conscious detection of emotional versus neutral facial expressions.

Third, the multiple regression analyses also revealed that EPN activity increased according to the level of emotional arousal. The most prevalent interpretation of dimensional emotional ratings is that arousal and valence reflect the intensity and quality of emotional experience, respectively (Lang et al., 1998 and Reisenzein, 1994). Based on this, our results indicate that EPN was enlarged because of their intense emotional significance. This is consistent with a recent ERP study reporting a positive relationship between EPN activity and arousal ratings for emotional scenes (Wiens et al., 2011), although the study used normative rating data in a different participant sample. We investigated the correspondence between emotional ratings and ERP activity in the same participants. The current study provides evidence that the increased EPN in response to emotional facial expressions reflects their emotional significance.

Taken together, our results regarding EPN suggest that the emotional versus neutral facial expressions activates the posterior visual cortices at $200-400 \mathrm{~ms}$, which is attributable not to the visual elements of emotional facial expressions but to their emotional significance. Our results revealed that increased EPN was related to faster and more accurate detection of the facial targets, and specifically to the higher emotional arousal felt by the participants. To our knowledge, this is the first evidence suggesting that the neural correlates of the efficient detection of emotional facial expressions are attributable to their emotional significance compared with emotionally neutral facial expressions when the physical changes in visual features are controlled.

The regression analysis showed a partial relationship between N2pc amplitude and detection RTs, showing larger N2pc for the face stimuli detected more rapidly at the right hemisphere. This indicates that the rapid detection of facial targets was accompanied by enhanced $\mathrm{N} 2 \mathrm{pc}$ amplitudes, which is consistent with previous studies showing that $\mathrm{N} 2 \mathrm{pc}$ was increased in response to emotional expressions that were detected more rapidly compared with those detected later (Feldmann-Wüstefeld et al., 2011 and Weymar et al., 2011). However, contrary to our prediction based on previous studies, repeated-measures ANOVA did not show that N2pc activity was modulated by the contrast between normal and anti-expressions. Several methodological differences, such as small visual eccentricities, different numbers of trials, and different electrode locations, may account for the disparity in the results. It is also possible that our control of visual factors may have produced the current results. Regarding this issue, a recent study tested the effect of the visual features (thickening the peripheral frames) of photos of 
emotional and neutral facial expressions on N2pc (Brosch et al., 2011). The results showed that exaggerated visual features increased N2pc activity, whereas such a difference was not observed in response to emotional versus neutral facial expressions. The researchers suggested that the neural mechanisms for visual and emotional attention may be dissociable (cf. Vuilleumier and Brosch, 2009) and that N2pc may reflect processing related to the former attentional system. Although some previous studies reported N2pc differences in response to photographs of negative and positive facial expressions after carefully matching the physical features (e.g., brightness) of these stimuli (Eimer and Kiss, 2007, Feldmann-Wüstefeld et al., 2011 and Fox et al., 2008), it is inevitable that different emotional expressions contain differing amounts of feature changes. We speculate that differences in N2pc may not be evident during the detection of emotional versus neutral facial targets when visual factors are controlled.

The regression analyses of the ERP data showed that EPN was not significantly related to the familiarity or naturalness ratings of facial targets. This suggests that these non-emotional processes of familiarity/naturalness cannot account for the EPN during the detection of facial expressions. However, our analyses of the behavioral data showed significant relationships between detection performance and these ratings, although the results were not consistent across the RT and accuracy measures. The results suggest the possibility that the processing of the familiarity and naturalness of facial expressions may influence the efficient detection of the expressions. Inspection of the rating patterns suggested that the effect of the familiarity/naturalness processes in response to faces was different from that of emotional arousal. However, additional behavioral and neuroscientific studies are needed to investigate the effects of these non-emotional processes on the detection of emotional facial expressions.

Regarding the difference between normal angry and happy expressions, our data assessing RTs and accuracy did not show significant differences in detection performance. This result differs from that of a previous study that used the same stimulus sets (Sato and Yoshikawa, 2010). Some methodological differences may account for the differing results. For example, we presented the stimuli only for $1000 \mathrm{~ms}$, whereas the previous study presented stimuli until participants completed their responses. Additionally, we presented the stimuli in a fixed position on the array, whereas the previous study presented the stimuli in random positions. However, other previous studies using photographic stimuli also reported mixed results regarding the more effective detection of angry versus happy expressions within an array of neutral faces; some reported positive (Fox and Damjanovic, 2006, Gilboa-Schechtman et al., 1999 and Lamy et al., 2008) and others reported negative (Byrne and Eysenck, 1995, Juth et al., 2005 and Williams et al., 2005) findings. Consistent with our behavioral data, the results of our ANOVA and regression analyses on EPN amplitude showed no effect related to emotional valence. These results are consistent with those of several previous studies showing no clear difference in EPN amplitude between negative and positive emotional scenes (e.g., Schupp et al., 2003; for a review, see Olofsson et al., 2008). We suggest that, as with behavioral performance, the differences in EPN between angry and happy expressions may be less robust than the differences between emotional and neutral facial expressions.

Some limitations to our study must be acknowledged. First, although the anti-expressions reflected changes in all facial features in equivalent amount but in the opposite directions from the emotional expressions with reference to the neutral expressions, this method cannot completely exclude the effects of all visual factors. It is possible that the efficient detection of facial expressions could be related to other visual factors, such as holistic information (cf. Tanaka and Farah, 1993) or interactions between features (cf. Itti and Koch, 2001). It was proposed 
that the recognition of facial expressions could be achieved by a holistic template matching strategy (e.g., Rutherford and McIntosh, 2007). Further studies are necessary to investigate the influence of these visual factors on the efficient detection of emotional facial expressions.

Second, only two models were used as stimuli because anti-expressions could not be created using facial materials with open mouths (Sato and Yoshikawa, 2009). Therefore, the present study could not assess differences between stimulus models such as sex, identity, and degree of distortion in the physical features. For example, a previous study reported that male angry faces were detected faster than female angry faces (Williams and Mattingley, 2006). Further investigation using more facial stimuli would allow these issues to be clarified.

Finally, because we recorded ERPs on the scalp, we failed to detect the activity of subcortical structures such as the amygdala. A previous ERP study suggested that the EPN for emotional facial expressions may be functionally related to subcortical activities (Sato et al., 2001). Some neuroimaging studies have reported that activity in the visual cortices in response to emotional facial expressions was modulated by amygdala activity (e.g., Morris et al., 1998). Other neuroimaging studies showed that amygdala activity while viewing emotional facial expressions reflected subjective emotional arousal/intensity (e.g., Sato et al., 2010). A recent intracranial EEG study has also shown that amygdala activation in response to emotional versus neutral facial expressions occurred rapidly, specifically during 50-150 ms (Sato et al., 2011). Taken together, these data suggest the possibility that the increased EPN in response to the detection of emotional versus neutral facial expressions found in the present study may be related to the rapid activation of the amygdala. In a future research, it would be interesting to investigate amygdala activity and its functional connectivity with the visual cortices during the detection of emotional facial expressions by using other neuroscientific measures such as functional magnetic resonance imaging and intracranial EEG.

In summary, our behavioral results showed that normal expressions were detected faster and more accurately than anti-expressions. Normal expressions, compared with anti-expressions, were also rated as more emotionally arousing. Our ERP results revealed that the normal expressions were associated with greater EPN. Furthermore, the enhanced EPN activity was related to shorter RTs, increased accuracy, and greater emotional arousal. Taken together, these multiple lines of behavioral and neuropsychological evidence suggest that the more rapid and more accurate detection of emotional than that of neutral facial expressions is implemented via activation of the posterior visual cortices at 200-400 ms based on their emotional significance.

\section{Experimental procedures}

\subsection{Participants}

Twenty volunteers (six females and 14 males, mean $\pm S D$ age, 23.1 \pm 3.6 ) participated. All participants gave written informed consent for the experimental procedure, which was approved by the ethics committee of the Primate Research Institute, Kyoto University. All participants were right handed as assessed by the Edinburgh Handedness Inventory ( Oldfield, 1971) and had normal or corrected-to-normal visual acuity.

\subsection{Experimental design}

The experiment consisted of target-present and target-absent trials. The target-present trials were constructed 
as a three-factor design with type (normal expression and anti-expression), emotion (anger and happiness), and visual field (the visual field in which the target was presented; left and right) as within-participant factors.

\subsection{Stimuli}

Target and distractor stimuli are shown in Fig. 1(a). Normal expressions and anti-expressions of angry and happy faces were used as target stimuli, and the neutral expressions were used as distractor stimuli.

Neutral expressions and normal emotional expressions depicting anger and happiness were grayscale photographs of two models (one female [PF] and one male [PE]) chosen from a standard set (Ekman and Friesen, 1976). Neither model was familiar to any of the participants. No expression showed bared teeth.

Anti-expressions were created from these photographs using computer-morphing software (FUTON System, ATR) on a Linux computer. First, the coordinates of 79 facial feature points were identified manually and realigned based on the coordinates of the bilateral irises. The differences between the points of the neutral and emotional expressions (angry and happy) were calculated and regarded as $0 \%$ and $100 \%$, respectively. The anti-expressions were then created by moving each feature point the same distance in the opposite direction with the emotional face, i.e. a $-100 \%$ transformation from the neutral expressions. Minor color adjustments by a few pixels were performed using Photoshop 5.0 (Adobe).

Two types of adjustments were made to the stimuli using PhotoShop 5.0. First, the photographs were cropped into a circle, slightly inside the frame of the face, to eliminate contours and hairstyles not relevant to the expression. Second, the photographs were prepared so that significant differences in contrast were eliminated, thereby removing possible identifying information.

To ensure that the stimuli could be recognized as facial expressions of the target emotion, we conducted a preliminary rating experiment with 18 participants (10 females and eight males), none of whom took part in the ERP experiment. We showed each facial expression of target and distractor stimuli and asked participants to select which of seven labels (anger, disgust, fear, happiness, sadness, surprise, and neutrality) most appropriately described the expression. The results showed that normal-angry, normal-happy, anti-angry, anti-happy, and neutral facial expressions were most frequently recognized as anger (52.8\%), happiness (83.3\%), neutrality (52.8\%), neutrality (41.7\%), and neutrality (86.1\%), respectively. Planned comparisons using $t$ values (one-tailed) showed that these labels were significantly more frequently selected than the other emotion labels for each facial expression $(t(119)>1.76, p<0.05)$. These results indicated that the normal and anti-expressions we used in the present experiment depicted emotional and neutral states, respectively.

\subsection{Procedure}

The presentation of stimuli was controlled by Presentation 14.9 (Neurobehavioral Systems) implemented on a Windows computer (HP Z200 SFF, Hewlett-Packard Company). The stimuli were presented on a 19-in. CRT monitor (HM903D-A, liyama) with a refresh rate $150 \mathrm{~Hz}$ and a resolution of $1024 \times 768$ pixels. The refresh rate was confirmed by using a high-speed camera (EXILIM FH100, Casio) with the temporal resolution of 1000 frames/s.

\subsubsection{Visual search task}

The stimuli were displayed in $2 \times 2$ arrays (four faces) against a black background. An example of the 
stimulus display is shown in Fig. 1(b). Each facial array was composed of pictures of a single model. Each individual face subtended a visual angle of $1.58^{\circ}$ horizontally $\times 1.93^{\circ}$ vertically, and the stimulus arrays subtended a visual angle of $4.30^{\circ} \times 4.30^{\circ}$. At the center of the stimulus arrays, a white cross subtending a visual angle of $0.86^{\circ} \times 0.86^{\circ}$ was presented as a fixation point.

The experiment consisted of a total of 768 trials presented in 12 blocks of 64 trials, with an equal number of target-present and target-absent trials. In the target-present trials, a discrepant picture was inserted into $2 \times 2$ arrays as the target. In the target-absent trials, each stimulus display consisted of the same four neutral faces. The trial number of each target condition was 96, with an equal number of targets presented in the left and right visual fields. The trial order was pseudo-randomized across all conditions within a block, although the order was restricted not to show the same target stimulus in the same position in successive trials. The interstimulus interval varied from 1300 to $1800 \mathrm{~ms}$. A white cross subtending a visual angle of $0.86^{\circ} \times 0.86^{\circ}$ was always displayed in the center of the monitor.

In each trial, the stimulus array consisting of four faces was presented for $1000 \mathrm{~ms}$. Participants were asked to answer as quickly and accurately as possible whether all four faces were the same (i.e., target-absent) or whether a discrepant face was present (i.e., target-present) by pushing the button on a response box (RB-530, Cedrus) using the left or right index finger. The position of the response buttons was counterbalanced across participants.

We measured ERPs during a visual search task. The experiment was conducted in an electrically shielded and soundproofed room (Science Cabin, Takahashi Kensetsu). Participants sat in chairs with their chins fixed in steady positions $80 \mathrm{~cm}$ from the monitor. They were asked to maintain their gaze on the fixation cross which was presented at the center of the display throughout the experiment. They were also instructed to avoid eye blinks and excessive bodily movements during trials. Before the experiment began, participants engaged in 32 practice trials to gain familiarity with the apparatus and the timing of eye blinks.

\subsubsection{Rating}

After ERP measurements during the visual search task, all facial stimuli were presented individually at random to the participants, who were asked to evaluate each stimulus in terms of the emotional arousal and valence experienced (i.e., the intensity and quality of the emotion that participants felt when perceiving the stimulus expression), familiarity (i.e., the frequency of seeing facial expressions in daily life such as those depicted by the stimulus), and naturalness (i.e., the degree of natural display of the stimulus expression). The ratings used a nine-point scale ranging from 1 (low arousal, negative, unfamiliar, and unnatural) to 9 (high arousal, positive, familiar, and natural). The order of presentation of the stimuli and rating items were randomized and balanced across participants.

\subsection{ERP measurement}

Electroencephalogram (EEG) signals were recorded with $28 \mathrm{Ag}-\mathrm{AgCl}$ passive sintered ring electrodes ( $\mathrm{Fp} 1$, Fp2, F7, F3, Fz, F4, F8, FC5, FC1, FCz, FC2, FC6, T7, C3, Cz, C4, T8, CP5, CP1, CP2, CP6, P7, P3, Pz, P4, P8, $\mathrm{O} 1$, and $\mathrm{O} 2$ ) mounted in an elastic cap (EasyCap) according to the modified international 10/20 system (Sharbrough et al. 1991). The cap was fixed with surgical tape under the jaw to keep the electrodes in position during the trials. Horizontal and vertical electrooculogram (EOG) signals were also recorded from the bilateral 
canthi and above and below the left eye. The reference electrode was put on the nose, and the ground electrode was fixed on the forehead (AFz electrode). Electrode impedance was kept below $15 \mathrm{k} \Omega$. EEG and EOG recordings were performed using a Brain Vision Recorder (Brain Products). Data were sampled at $500 \mathrm{~Hz}$ and filtered through a band-pass of 0.016-250 Hz. An unobtrusive monitoring of events was also made using a video camera (SA-48825, Entel System) to check participants' eye and body movements.

\subsection{Data analysis}

\subsubsection{Behavioral data}

The mean RTs of the correct responses and mean accuracy in target trials were calculated for each target stimulus condition, excluding trials beyond mean $\pm 3 S D$ of RTs as artifacts. The RTs and accuracy rate were then subjected to three-way repeated-measure ANOVA with type, emotion, and visual field of target presentation as within-participant factors. For significant interactions, follow-up analyses of the simple effect were conducted (Kirk, 1995). When interactions were significant, main effects were not subjected to interpretation because of the possibilities of problems (cf. Tabachnick and Fidell, 2000).

Each rating (arousal, valence, familiarity, and naturalness) for target stimuli was analyzed using two-way repeated-measures ANOVA with type and emotion as within-participant factors. For significant interactions, as with the RT and accuracy data, follow-up analyses were conducted.

Multiple regression analysis was performed with the single rating as an independent variable and RT or accuracy averaged across the left and right visual fields under all typexemotion conditions as a dependent variable. Dummy variables were added as independent variables to represent participant as a factor of no interest.

All statistical tests for the behavioral data were performed using SPSS 10.0J software (SPSS Japan), and the results were deemed statistically significant at $p<0.05$.

\subsubsection{ERP}

ERP analyses were performed using SPM8 (http://www.fil.ion.ucl.ac.uk/spm/software/spm8) and implemented using Matlab R2011b (Mathworks). Only the data in target-present trials with correct responses and RTs within $3 S D$ were analyzed. The ERP and EOG data obtained during $1200 \mathrm{~ms}$ were sampled at a rate of $500 \mathrm{~Hz}$ for each trial; prestimulus baseline data were collected for $200 \mathrm{~ms}$ (while the fixation point was presented), and experimental data were collected for $1000 \mathrm{~ms}$ after stimulus onset. The data were subjected to topography-based correction using the Berg algorithm ( Berg and Scherg, 1994) for the purpose of eye-movement rejection. Data were filtered offline through a band-pass of $0.1-30 \mathrm{~Hz}$ in the forward and reverse directions using a fifth-order Butterworth digital filter and then baseline corrected relative to the $200 \mathrm{~ms}$ before stimulus. Trials with either $\geq 75 \mu \mathrm{V}$ in EEG or $\geq 50 \mu \mathrm{V}$ in EOG were rejected as artifacts and excluded from further analyses. The mean $\pm S D$ frequencies of artifact-contaminated trials were $26.3 \pm 11.8 \%$ and were not different across conditions $(p>0.10)$ using three-way repeated-measures ANOVA. The ERP data were then averaged over trials by condition.

ERP data were analyzed using a 3D space-time SPM approach (Kilner and Friston, 2010 and Litvak et al., 2011). The advantages of this approach are that it allows for the spatio-temporal localization of effects while controlling for multiple comparisons over latencies and/or electrodes; the analysis for each time bin in this approach can also be more sensitive than conventional averaging (Kilner and Friston, 2010). First, the averaged 
ERP data were converted to images in three dimensions (i.e., space ( $x$ and $y$ ) and time (z)), by creating a two-dimensional linearly interpolated scalp topographic map for each time bin and stacking scalp maps over peristimulus time. The resulting 3D space-time images contained spatial and time dimensions of $32 \times 32$ pixels and 1200 time bins.

Then, these space-time images were entered into random-effects general linear models (GLMs). We constructed two types of GLMs. First, to accomplish a repeated-measures ANOVA contrasting normal versus anti-expressions, we constructed a GLM including type, emotion, and visual field as factors of interest, which compare EPN/N2pc among target stimulus conditions. Next, to conduct multiple regression analyses regarding the relationships between ERP and behavioral data (RT, accuracy or ratings), we constructed GLMs including the single behavioral measure under all typexemotion conditions as a factor of interest and participant as a factor of no interest. This revealed the relationship between the EPN/N2pc activities and detection performance or ratings. This method can estimate the contribution of ERP activities to detection performance or emotional ratings directly (cf. Wiens et al., 2011). To analyze the relationships between the EPN activities and detection performance/ratings, the $\mathrm{ERP}$ and RT data of the left and right visual fields were averaged. To analyze the relationships between N2pc amplitude and RT/ratings, differences in ERP and RT/accuracy contralateral and ipsilateral to a target were computed. According to a previous ERP study (Weymar et al., 2011), the ipsilateral waveform was defined as the averaged waveform at the P7 electrode when the target was presented to the left visual field and that at the P8 electrode when the target was presented to the right visual field. The contralateral waveform was defined as the averaged waveform at the P7 electrode when the target was presented to the right visual field and that at the P8 electrode when the target was presented to the left visual field.

For the repeated-measures ANOVA, corrections for non-sphericity were applied to ensure the assumption of an independent and identically distributed error for the GLM. We modeled the covariance components representing dependency and uneven variance between levels. Covariance components were estimated from the pooled active voxels (exceeding an uncorrected $F$ threshold of $p<0.01$ for any effect) with the restricted maximum-likelihood procedure ( Friston et al., 2002). The inverse of the square root of the estimated covariance matrix was used to prewhiten the data and design matrix. A least-squares estimation was performed on the whitened data and design matrix, giving the maximum-likelihood parameter estimates.

Finally, space-time SPM $\{T\}$ images were generated for each contrast according to our predictions. To test our specific predictions for EPN/N2pc, we used the restricted space-time window for the space-time ROI. This approach is equivalent to the small volume correction in a standard SPM (Worsley et al., 1996). We defined the ROI as the P7 and P8 electrode locations (bilateral for EPN and contralateral for N2pc) during 200-400 ms because previous ERP studies showed EPN/N2pc activities at these electrode sites (e.g., Feldmann-Wüstefeld et al., 2011 and Sato et al., 2001) that were recorded around 200-400 ms after stimulus onset with a peak around $300 \mathrm{~ms}$ (e.g. Feldmann-Wüstefeld et al., 2011 and Schupp et al., 2004). For repeated-measures ANOVA, to clarify the effect of emotional significance on ERP components, we tested the main effect of type (normal versus anti-expression) for EPN. We also tested the interaction between type (normal versus anti-expression) and visual field (left versus right) for N2pc at the P7 and P8 electrodes respectively to examine a three-way interaction among type, visual field of target presentation, and electrode site. For the regression analyses, we tested the effect of 
behavioral data. To test the effects without predictions, the entire scalp and time ( -200 to $1000 \mathrm{~ms}$ ) regions were analyzed. Significant activation was identified if the activation foci reached the height threshold of $p<0.05$, family-wise error corrected for multiple comparisons, based on the random field theory ( Kilner et al., 2005 and Worsley et al., 1996). The equivalent $Z$-value was used to report the inferential results. To report the time extent of the activation clusters, including the significantly activated foci, the space-time $\operatorname{SPM}\{T\}$ was thresholded at a height threshold of uncorrected $p<0.01$. We also conducted some analyses with more liberal thresholds for descriptive purposes.

Additionally, we conducted ROI analyses for the mean amplitudes at 200-400 ms for target-present trials to confirm and complement the results of the bin-based SPM approach. A four-way ANOVA with type (normal and anti-expression), emotion (anger and happiness), visual field (left and right), and electrode (P7 and P8) as factors was conducted to confirm the effect of type on EPN and N2pc. To test the activities at adjacent electrodes, the ERP data at O1/O2 and P3/P4 were also analyzed in the same way. Regression analyses were also conducted to confirm the relationship between EPN/N2pc and behavioral performance or ratings. The mean ERP data across the electrode sites were analyzed, after the data were calculated by averaging between left and right visual field for EPN analyses, and by subtracting ipsilateral from contralateral for N2pc analyses.

\section{Acknowledgments}

This work was supported by funds from the Japan Society for the Promotion (JSPS) of Science Funding Program for Next Generation World-Leading Researchers (NEXT Program) no. LZ008 and JSPS KAKENHI Grant no. 25885049 .

\section{References}

Balconi, M., Pozzoli, U., 2003. Face-selective processing and the effect of pleasant and unpleasant emotional expressions on ERP correlates. Int. J. Psychophysiol. 49, 67-74.

Berg, P., Scherg, M., 1994. A multiple source approach to the correction of eye artifacts. Electroencephalogr. Clin. Neurophysiol. 90, 229-241.

Brosch, T., Pourtois, G., Sander, D., Vuilleumier, P., 2011. Additive effects of emotional, endogenous, and exogenous attention: behavioral and electrophysiological evidence. Neuropsychologia 49, 1779-1787.

Byrne, A., Eysenck, M.W., 1995. Trait anxiety, anxious mood, and threat detection. Cogn. Emot. 9, 549-562.

Cave, K.R., Batty, M.J., 2006. From searching for features to searching for threat: drawing the boundary between preattentive and attentive vision. Vis. Cogn. 14, 629-646.

Darwin, C., 1872. In: The Expression of the Emotions in Man and Animal third ed. Oxford University Press, New York, NY.

Eimer, M., Kiss, M., 2007. Attentional capture by task-irrelevant fearful faces is revealed by the N2pc component.

Biol. Psychol. 74, 108-112

Ekman, P., Friesen, W.V., 1976. In: Pictures of Facial Affect.Consulting Psychologists Press, Palo Alto, CA.

Feldmann-Wustefeld, T., Schmidt-Daffy, M., Schubö, A., 2011. Neural evidence for the threat detection advantage: differential attention allocation to angry and happy faces.Psychophysiology 48, 697-707. 
Fox, E., Damjanovic, L., 2006. The eyes are sufficient to produce a threat superiority effect. Emotion 6, 534-539.

Fox, E., Derakshan, N., Shoker, L., 2008. Trait anxiety modulates the electrophysiological indices of rapid spatial orienting towards angry faces. NeuroReport 19, 259-263.

Friston, K.J., Glaser, D.E., Henson, R.N., Kiebel, S., Phillips, C., Ashburner, J., 2002. Classical and Bayesian inference in neuroimaging: applications. NeuroImage 16, 484-512.

Genetti, M., Khateb, A., Heinzer, S., Michel, C.M., Pegna, A.J., 2009. Temporal dynamics of awareness for facial identity revealed with ERP. Brain Cogn. 69, 296-305.

Gilboa-Schechtman, E., Foa, E.B., Amir, N., 1999. Attentional biases for facial expressions in social phobia: the face-in-the-crowd paradigm. Cogn. Emot. 13, 305-318.

Hansen, C.H., Hansen, R.D., 1988. Finding the face in the crowd: an anger superiority effect. J. Per sonal. Soc. Psychol. 54, 917-924.

Itti, L., Koch, C., 2001. Computational modelling of visual attention. Nat. Rev. Neurosci. 2, 194-203.

Junghöfer, M., Bradley, M.M., Elbert, T.R., Lang, P.J., 2001. Fleetingimages: a new look at early emotion discrimination. Psychophysiology 38, 175-178.

Juth, P., Lundqvist, D., Karlsson, A., Öhman, A., 2005. Looking for foes and friends: perceptual and emotional factors when finding a face in the crowd. Emotion 5, 379-395.

Kilner, J.M., Friston, K.J., 2010. Topological inference for EEG and MEG. Ann. Appl. Stat. 4, 1272-1290.

Kilner, J.M., Kiebel, S.J., Friston, K.J., 2005. Applications of random field theory to electrophysiology. Neurosci. Lett. 374, 174-178.

Kirk, R.E., 1995. In: Experimental Design: Procedures for the Behavioral Sciences. Brooks/Cole Publishing Company, Pacific Grove, CA.

Koivisto, M., Revonsuo, A., 2010. Event-related brain potential correlated of visual awareness. Neurosci. Biobehav. Rev. 34, 922-934.

Lamy, D., Amunts, L., Bar-Haim, Y., 2008. Emotional priming of pop-out in visual search. Emotion 8, 151-161.

Lang, P.J., Bradley, M.M., Cuthbert, B.N., 1998. Emotion and motivation: measuring affective perception. J. Clin. Neurophysiol. 15, 397-408.

Litvak, V., Mattout, J., Kiebel, S.J., Phillips, C., Henson, R.N.A., Kilner, J., et al., 2011. EEG and MEG data analysis in SPM8. Comput. Intell. Neurosci. 15, 1-32.

Luck, S.J., Hillyard, S.A., 1994. Electrophysiological correlates of feature analysis during visual search. Psychophysiology 31, 291-308.

Morris, J.S., Friston, K.J., Buchel, C., Frith, C.D., Young, A.W., Calder, A.J., et al., 1998. A neuromodulatory role for the human amygdala in processing emotional facial expressions. Brain 121, 47-57.

Öhman, A., Lundqvist, D., Esteves, F., 2001. The face in the crowd revisited: a threat advantage with schematic stimuli. J. Personal. Soc. Psychol. 80, 381-396.

Oldfield, R.C., 1971. The assessment and analysis of handedness: the Edinburgh inventory. Neuropsychologia 9, 97-113.

Olofsson, J.K., Nordin, S., Sequeira, H., Polich, J., 2008. Affective picture processing: an integrative review of ERP findings. Biol. Psychol. 77, 247-265. 
Reisenzein, R., 1994. Pleasure-arousal theory and the intensity ofemotions. J. Personal. Soc. Psychol. 67, 525-539.

Rutherford, M.D., McIntosh, D.N., 2007. Rules versus prototype matching: strategies of perception of emotional facial expressions in the autism spectrum. J. Autism Dev. Disord. 37, 187-196.

Sagi, D., Julesz, B., 1986. Enhanced detection in the aperture of focal attention during simple discrimination tasks. Nature 321, 693-695.

Sato, W., Kochiyama, T., Uono, S., Matsuda, K., Usui, K., Inoue, Y., et al., 2011. Rapid amygdala gamma oscillations in response to fearful facial expressions. Neuropsychologia 49, 612-617.

Sato, W., Kochiyama, T., Yoshikawa, S., 2010. Amygdala activity in response to forward versus backward dynamic facial expressions. Brain Res. 1315, 92-99.

Sato, W., Kochiyama, T., Yoshikawa, S., Matsumura, M., 2001. Emotional expression boosts early visual processing of the face: ERP recording and its decomposition by independent component analysis. NeuroReport 12, 709-714.

Sato, W., Yoshikawa, S., 2009. Anti-expressions: artificial control stimuli for the visual properties of emotional facial expressions. Soc. Behav. Personal. 37, 491-502.

Sato, W., Yoshikawa, S., 2010. Detection of emotional facial expressions and anti-expressions. Vis. Cogn. 18, 369-388.

Schupp, H.T., Junghöfer, M., Öhman, A., Weike, A.I., Stockburger, J., Hamm, A.O., 2004. The facilitated processing of threatening faces: an ERP analysis. Emotion 4, 189-200.

Schupp, H.T., Junghöfer, M., Weike, A.I., Hamm, A.O., 2003. Emotional facilitation of sensory processing in the visual cortex. Psychol. Sci. 14, 7-13.

Sharbrough, F., Chatrian, G.-E., Lesser, R.P., Lers, H., Nuwer, M., Picton, T.W., 1991. American electroencephalographic society guidelines for standard electrode position nomenclature. J. Clin. Neurophysiol. $8,200-202$.

Skinner, A.L., Benton, C.P., 2010. Anti-expression aftereffects reveal prototype-referenced coding of facial expressions. Psychol. Sci. 21, 1248-1253.

Skinner, A.L., Benton, C.P., 2012. Visual search for expressions and anti-expressions. Vis. Cogn. 20, 1186-1214.

Tabachnick, B.G., Fidell, L.S., 2000. In: Computer-assisted Research Design and Analysis. Allyn and Bacon, Boston, MA.

Tanaka, J.W., Farah, M.J., 1993. Parts and wholes in face recognition. Q. J. Exp. Psychol. A 46, 225-245.

Tong, F., Nakayama, K., 1999. Robust representations for faces: evidence from visual search. J. Exp. Psychol.: Hum. Percept. Perform. 25, 1016-1035.

Vuilleumier, P., Brosch, T., 2009. Interactions of emotion and attention in perception. In: Gazzaniga, M.S. (Ed.), The Cognitive Neurosciences fourth ed. MIT Press, Cambridge, MA, pp. 925-934.

Weymar, M., Löw, A., Ohman, A., Hamm, A.O., 2011. The face is more than its parts - brain dynamics of enhanced spatial attention to schematic threat. NeuroImage 58, 946-954.

Wiens, S., Sand, A., Olofsson, J.K., 2011. Nonemotional features suppress early and enhance late emotional electrocortical responses to negative pictures. Biol. Psychol. 86, 83-89.

Williams, M.A., Mattingley, J.B., 2006. Do angry men get noticed?. Curr. Biol. 16, R402-R404. 
Williams, M.A., Moss, S.A., Bradshaw, J.L., Mattingley, J.B., 2005. Look at me, I'm smiling: visual search for threatening and nonthreatening facial expressions. Vis. Cogn. 12, 29-50.

Woodman, G.F., Luck, S.J., 1999. Electrophysiological measurement of rapid shifts of attention during visual search. Nature 400, 867-869.

Worsley, K.J., Marrett, S., Neelin, P., Vandal, A.C., Friston, K.J., Evans, A.C., 1996. A unified statistical approach for determining significant signals in images of cerebral activation. Hum. Brain Mapp. 4, 58-73. 